\title{
$\beta$-catenin induces A549 alveolar epithelial cell mesenchymal transition during pulmonary fibrosis
}

\author{
PING SONG ${ }^{1}$, JIN-XU ZHENG ${ }^{1}$, JIAO XU ${ }^{1}$, JI-ZHU LIU ${ }^{2}$, LI-YAN WU $^{1}$ and CHAO LIU ${ }^{1}$ \\ ${ }^{1}$ Respiratory Department, Affiliated Hospital of Jiangsu University, Zhenjiang, Jiangsu 212001; \\ ${ }^{2}$ Respiratory Department, Huaibei Miner General Hospital, Huaibei, Anhui 235000, P.R. China
}

Received December 23, 2013; Accepted October 24, 2014

DOI: $10.3892 / \mathrm{mmr} .2014 .3013$

\begin{abstract}
Idiopathic pulmonary fibrosis (IPF) is a fatal lung disease characterized by expansion of the fibroblast and myofibroblast population and extracellular matrix deposition. Although the pathogenic mechanisms of IPF remain to be fully elucidated, there is emerging evidence that fibroblasts and myofibroblasts may be derived partially from alveolar epithelial cells by epithelial-mesenchymal transition (EMT). In the present study, A549 cells were treated with different concentrations of Wntl and the results indicated that the mRNA and protein expression levels of vimentin, $\alpha$-smooth muscle actin ( $\alpha$-SMA) and collagen I gradully increased and those of E-cadherin gradully decreased in a concentration-dependent manner. Furthermore, the A549 cells were transfected with $\beta$-catenin plasmid cells, revealing phenotypic changes in the cells from a pebble to a fusiform shape. The mRNA and protein expression levels of of vimentin, $\alpha$-SMA and collagen I increased significantly, whereas those of E-cadherin decreased significantly. The present study examined the roles of alveolar epithelial cell injury and profibrogenic cytokine release in EMT and their association with the Wnt/ $\beta$-catenin signaling pathway in a mouse model of bleomycin-induced pulmonary fibrosis. Bronchoalveolar fluid was obtained 7 days after treatment with bleomycin and the A549 cells were incubated for $48 \mathrm{~h}$. An increase in the expression levels of the mesenchymal markers, $\alpha$-SMA, vimentin and collagen I, and a concomitant decrease in the expression of the epithelial marker, E-cadherin were observed. This corresponded with an increased expression of $\beta$-catenin. When the A549 cells were infected with a lentivirus expressing $\beta$-catenin shRNA, no significant increase was observed in the expression of the mesenchymal cell markers and the expression of E-cadherin did not decrease. These findings demonstrated that activation of the Wnt signaling pathway was
\end{abstract}

Correspondence to: Professor Jin-Xu Zheng, Respiratory Department, Affiliated Hospital of Jiangsu University, 438 Jiefang Road, Zhenjiang, Jiangsu 212001, P.R. China

E-mail: jxuzh135@163.com

Key words: epithelial-mesenchymal transition, $\beta$-catenin, fibroblasts, lung epithelium capable of inducing an EMT program in the lung epithelial cells through $\beta$-catenin and that injured alveolar epithelium activated the Wnt $/ \beta$-catenin signaling pathway, thereby inducing the expansion of the fibroblast/myofibroblast population through EMT. These results suggested that $\beta$-catenin was involved in the formation of lung fibrosis and may provide a theoretical basis for the treatment of IPF.

\section{Introduction}

Idiopathic pulmonary fibrosis (IPF) is a progressive disorder of unknown etiology, which has limited response to currently available therapies and a mean survival expectancy of 3-5 years (1). Over the last few years, there has been increasing evidence that IPF may result from acute lung injury targeting alveolar epithelial cells and consequent aberrant wound healing leading to the formation of fibroblastic foci, which are considered to be the active sites of fibrogenesis (2). Fibroblastic foci are composed mainly of fibroblasts and myofibroblasts, which promote excessive deposition of extracellular connective matrix in the pulmonary interstitium during the pathogenesis and progression of pulmonary fibrosis, which results in irreversible distortion of the lung architecture (3-5).

The origin of fibroblasts and myofibroblasts in these fibroblastic foci remain to be fully elucidated, although the migration and proliferation of resident mesenchymal cells and recruitment of fibrocytes may account for a fraction of them. In 2005, Willis et al identified commonly expressed epithelial markers and $\alpha$-smooth muscle actin ( $\alpha$-SMA) in the lung tissues of patients with IPF, indicating that cells undergo phenotypic transition in the IPF lung and describing, for the first time, the possibility of epithelial-mesenchymal transition (EMT) in human lung fibrosis (4). There has been increasing evidence that lung fibroblasts and myofibroblasts may be derived from epithelial cells through EMT (6-8). EMT is crucial for germ layer formation and cell migration in the early vertebrate embryo (9). Although EMT is usually maintained in a silent state in adults, it may be transiently activated for wound healing and tissue repair $(9,10)$ and there is increasing evidence that abnormal activation of EMT programs are associated with tissue fibrosis (11). EMT is characterized by morphological changes, including the change from a cuboidal cell shape to an elongated or spindle-shaped form, acquisition of fibroblast- or myofibroblast-specific markers vimentin, 
collagen fiber I and $\alpha$-SMA, loss of the characteristic epithelial marker E-cadherin and epithelial cell polarity, abatement of adhesion ability and cytoskeletal rearrangements $(10,12)$. The mechanisms of EMT, however, remain to be elucidated. Several studies have demonstrated that multiple cytokines are effected in EMT, including tumor growth factor- $\beta$, insulin-like growth factor-II and fibroblast growth factor-2 (4,13-15).

Wnt signals are important in embryonic development and organ morphogenesis. Previous studies have demonstrated that abnormal activation of the $\mathrm{Wnt} / \beta$-catenin signaling pathway occurs in the lung tissue of patients with IPF and in models of bleomycin-induced pulmonary fibrosis $(16,17)$. However, the precise mechanism through which this occurs remains unclear. Wnt signaling cascades can be divided into at least three distinct pathways, one of which is the Wnt/ $\beta$-catenin signaling pathway. This classical signaling pathway is initiated by extracellular ligands, termed Wnts. $\beta$-catenin is the key member of the Wnt signaling pathway in the regulation of transcriptional activity (18). In the present study, A549 cells were treated with different concentrations of Wnt1 and transfected with a $\beta$-catenin plasmid, the results indicated that Wnt/ $\beta$-catenin led to activation of an EMT transcriptome. Previously, a model has been proposed in which injury to the epithelium initiates a proinflammatory and profibrotic cascade resulting in fibroblast expansion and progressive fibrosis reminiscent of abnormal wound healing (19). The present study investigated whether injured alveolar epithelia induce EMT and activate the Wnt/ $\beta$-catenin signal pathway. The A549 cells were cultured with bronchoalveolar lavage fluid (BALF) from bleomycin-treated mice in the presence or absence of a small interfering (si)RNA designed to suppress the expression of $\beta$-catenin.

\section{Materials and methods}

Cell culture. A549, human alveolar epithelial cells (American Type Culture Collection, Manassas, VA, USA), were purchased from the Institute of Biochemistry and Cell Biology (Shanghai Institute of Biological Science, Chinese Academy of Sciences, Shanghai, China) and maintained in Dulbecco's modified Eagle's medium (DMEM; Sunshine Biotechnology Co., Ltd, Jiangsu, China) supplemented with $10 \%$ fetal bovine serum (FBS; Invitrogen Life Technologies, Carlsbad, CA, USA) at $37^{\circ} \mathrm{C}$ in a humidified $5 \% \mathrm{CO}_{2}$ atmosphere. The $\mathrm{A} 549$ cells were diluted with DMEM containing $10 \% \mathrm{FBS}$ to $1 \times 10^{5} \mathrm{cells} / \mathrm{ml}$ and seeded into six-well plates $(2 \mathrm{ml} /$ well; Corning Inc., Corning NY, USA). When cells reached $60-70 \%$ confluence the culture medium was replaced with $2 \mathrm{ml}$ serum-free DMEM for $24 \mathrm{~h}$ prior to treatment.

$B A L F$. In the present study, 6-8-week-old (18 \pm 2 g) Specific pathogen-free female C57BL/6 mice (Laboratory Animal Center of Jiangsu University, Jiangsu, China) were used. The animal experiments were approved by the Animal Research Committee of Jiangsu University School of Medicine (Jiangsu, China) and clean food and water were provided ad libitum. The BALF procedure was performed on day 7 following intra-tracheal injection of bleomycin solution $(5 \mathrm{mg} / \mathrm{kg}$ body weight; Nippon Kayaku Co., Ltd, Tokyo, Japan), as previously described (20-22). Briefly, on day 7 post-modeling, the mice were sacrificed by cervical dislocation and, following excision of the trachea, a plastic cannula was inserted into the trachea and $1.0 \mathrm{ml}$ cold sterile saline solution was injected gently with a syringe and withdrawn. This procedure was repeated three times. The BALF was then centrifuged for $5 \mathrm{~min}$ at $716 \mathrm{xg}$ and the supernatants were preserved at $-70^{\circ} \mathrm{C}$. Subsequently, the lung tissues were harvested.

Hematoxylin and eosin $(H \& E)$ staining. The mice were sacrificed and the lungs were rinsed in phosphate-buffered saline (Huashun Biotechnology Co., Ltd, Shanghai, China) fixed in $4 \%$ paraformaldehyde for $24 \mathrm{~h}$, embedded in paraffin and sectioned at $5 \mu \mathrm{M}$. H\&E staining was performed for cell alignment to evaluate the degrees of inflammation.

Cell transfection with plasmids. Plasmids expressing constitutively active $\beta$-catenin (plasmid pcDNA DEST40) were obtained from Shanghai Integrated Biotech Solutions Co, Ltd. (Shanghai, China). When the A549 cells reached 60-70\% confluence on six-well plates, they were transfected using Lipofectamine 2000 (Invitrogen Life Technologies), according to manufacturer's instructions. Subsequently, $2 \mathrm{~h}$ prior to transfection, the medium was replaced with serum-free DMEM and the $\beta$-catenin plasmid and Lipofectamine were then diluted separately in $250 \mu$ l Opti-MEM (Invitrogen Life Technologies). Subsequently, $6 \mu \mathrm{g}$ plasmid DNA per well was complexed with $4 \mu 1$ Lipofectamine. The complexes were then incubated at room temperature for $20 \mathrm{~min}$ and added to the cells in six-well plates. Following $4 \mathrm{~h}$ incubation, the cell medium was replaced by fresh DMEM with $10 \%$ FBS and the cells were incubated for a further for $48 \mathrm{~h}$. Empty plasmids were used as controls.

Transfection of lentiviral vectors with shRNA for $\beta$-catenin. Stealth small interference (si)RNA sequences for $\beta$-catenin were designed by Shanghai Integrated Biotech Solutions Co, Ltd. The pLentilox3.7-GFP-shRNA- $\beta$-catenin lentiviral vectors were synthesized using the following target shRNA sequence: 5'-CAGTTGTGGTTAAGCTCTT-3'. An unrelated shRNA sequence was used as a negative control (shNC): 5'-TTCTCCGAACGTGTCACGT-3'. The lentiviral vectors and lentiviral helper plasmids (VSVG, RSV-REV and pMDLg/pRRE) were also cotransfected into the $293 \mathrm{~T}$ cells. At 48 and $72 \mathrm{~h}$ post-co-transfection, the culture media were colleted and centrifuged for $20 \mathrm{~min}$ at 1,600 $\mathrm{x}$ g. The supernatants were filtered through a Millex-HV polyvinylidene fluoride- $0.45 \mu \mathrm{m}$ filter (Millipore, Billerica, MA, USA). The flow-through containing the virus was stored at $-70^{\circ} \mathrm{C}$ until further use as a viral stock. The A549 cells were cultured to $40-50 \%$ confluence and then infected with either the lentivirus expressing a shRNA to the human $\beta$-catenin gene (sh $\beta$-catenin) or with the negative control plasmid at a multiplicity of infection of 20. The number of green fluorescent protein (GFP)-positive cells was determined using an inverted fluorescent microscope (Axio Observer; magnification, x20; Carl Zeiss, Oberkochen, Germany) 4 days post-transduction to evaluate the transfection efficiency. Validation of the shRNA targeting sequence with the most efficient interference with $\beta$-catenin was then performed by 
Table I. RT-qPCR primers, conditions and products.

\begin{tabular}{|c|c|c|c|c|}
\hline RT-qPCR genes & S/AS & Primer sequence $\left(5^{\prime}-3^{\prime}\right)$ & Temperature $\left({ }^{\circ} \mathrm{C}\right)$ & Product (bp) \\
\hline$\alpha$-SMA & $\begin{array}{l}\mathrm{S} \\
\mathrm{AS}\end{array}$ & $\begin{array}{l}\text { 5'-TCAAATACCCCATTGAACACGG-3' } \\
\text { 5'-GGTGCTCTTCAGGTGCTACA-3' }\end{array}$ & 58 & 178 \\
\hline Vimentin & $\begin{array}{l}\text { S } \\
\text { AS }\end{array}$ & $\begin{array}{l}\text { 5'-TGCGTGAAATGGAAGAGAACT-3' } \\
\text { 5'-TGCGTGAAATGGAAGAGAACT-3' }\end{array}$ & 58 & 240 \\
\hline Collagen I & $\begin{array}{l}\mathrm{S} \\
\mathrm{AS}\end{array}$ & $\begin{array}{l}\text { 5'-TCTGACTGGAAGAGTGGAGAGTAC-3' } \\
\text { 5'-ATCCATCGGTCATGCTCTCG-3' }\end{array}$ & 58 & 202 \\
\hline E-cadherin & $\begin{array}{l}\mathrm{S} \\
\mathrm{AS}\end{array}$ & $\begin{array}{l}\text { 5'-TTGCTACTGGAACAGGGACAC-3' } \\
\text { 5'-CCCGTGTGTTAGTTCTGCTGT-3' }\end{array}$ & 58 & 179 \\
\hline$\beta$-catenin & $\begin{array}{l}\mathrm{S} \\
\mathrm{AS}\end{array}$ & $\begin{array}{l}\text { 5'-GCTACTCAAGCTGATTTGATGGA-3' } \\
\text { 5'-GGTAGTGGCACCAGAATGGATT-3' }\end{array}$ & 58 & 120 \\
\hline GAPDH & $\begin{array}{l}\text { S } \\
\text { AS }\end{array}$ & $\begin{array}{l}\text { 5'-GGATTTGGTCGTATTGGG-3' } \\
\text { 5'-GGAAGATGGTGATGGGATT-3' }\end{array}$ & 58 & 205 \\
\hline
\end{tabular}

$\mathrm{S}$, sense; AS, antisense; RT-qPCR, reverse transcription-quantitative polymerase chain reaction; $\alpha$-SMA, $\alpha$-smooth muscle actin.

reverse transcription quantitative polymerase chain reaction (RT-qPCR) and western blot analysis.

Western blotting. Cells were lysed in an ice-cold radioimmunoprecipitation assay lysis buffer [50 mM Tris ( $\mathrm{pH} 7.4)$, $150 \mathrm{mM} \mathrm{NaCl}, 1 \%$ Triton X-100, $1 \%$ sodium deoxycholate and $0.1 \%$ sodium dodecyl sulfate (SDS)]. Equal quantities of protein (20 $\mu \mathrm{g} /$ lane) were resolved on a $12 \%$ SDS-polyacrylamide gel. The proteins were then transferred onto polyvinylidene fluoride membranes (Millipore, Billerica, MA, USA and Weiga Science and Technology Co., Ltd, Guangzhou, China), inhibited with skimmed milk and probed using mouse anti-human monoclonal antibodies against $\alpha$-SMA (sc-53015), vimentin (sc-373717) and $\beta$-actin (sc8432; 1:1,000; Santa Cruz Biotechnology, Inc., Santa Cruz, CA, USA), rabbit anti-human polyclonal antibodies against collagen I (sc-28657; 1:1,000; Santa Cruz Biotechnology, Inc.), E-cadherin (BA0475; 1:200; Boster Biological Technology, Wuhan, China) or $\beta$-catenin (9562; 1:1,000; Cell Signaling Technology, Inc., Boston, MA, USA), followed by horseradish peroxidase-conjugated goat anti-rabbit immunoglobulin G (sc-2004) and goat anti-mouse antibodies (sc-2005; 1:5,000; Santa Cruz Biotechnology, Inc.). Enhanced chemiluminescence detection reagents were used for visualization (Amersham Pharmacia Biotech, Piscataway, NJ, USA) and the band densities for each phenotype marker were quantified using Lane 1D software (version 2.0; Beijing Sage Creation Science Co., Ltd., Beijing, China) following scanning with an ECL-PLUS chemiluminescence system (Bio-Rad, Hercules, CA, USA). $\beta$-actin staining served as an internal control and the ratio of band density to total $\beta$-actin was determined.

$R T$-qPCR. Total RNA was isolated using TRIzol ${ }^{\circledR}$ reagent (Invitrogen Life Technologies) according to the manufacturer's instructions and cDNAs were generated using a PrimeScript RT reagent kit (Takara Bio, Inc., Dalian, China). qPCR were performed using an Mx3000P system (Stratagene,
La Jolla, CA, USA) with SYBR Premix Ex Taq (Takara Bio, Inc.). The primers and conditions for $\mathrm{qPCR}$ are detailed in Table I. The RT reaction mixture $(1 \mu \mathrm{l})$ was used for the qPCR reaction in a total volume of $20 \mu \mathrm{l}$. The relative transcript abundance of a gene was presented as the $\Delta \mathrm{Ct}$ values $\left(\Delta \mathrm{Ct}=\mathrm{Ct}^{\text {reference }}-\mathrm{Ct}^{\text {target }}\right)$ and the relative expression levels of the target genes, following normalization to the endogenous sequence, were calculated using the $2^{-\Delta \Delta \mathrm{Ct}}$ method.

Statistical analysis. The results are expressed as the mean \pm standard deviation. Statistical comparisons between the groups were performed using a two-tailed unpaired t-test or one-way analysis of variance followed by a Student-Newman-Keuls-q test for analysis of more than two groups. Correlation analysis adopted Pearson's correlation analysis using SPSS for Windows, version 16.0 (SPSS, Inc., Chicago, IL, USA). P<0.05 was considered to indicate a statistically significant difference.

\section{Results}

Effects on the mRNA and protein expression levels of E-cadherin, SMA, vimentin and collagen I in A549 cells stimulated by Wnt1. The A549 cells were stimulated with various concentrations of Wnt1 $(0,5,10,20$ and $40 \mu \mathrm{g} / \mathrm{l})$ for $48 \mathrm{~h}$. Western blot analysis and RT-qPCR revealed that the mRNA and protein expression of levels E-cadherin decreased and the mRNA and protein expression levels of SMA, vimentin and collagen I increased in a concentration-dependent manner, with Wnt1 concentration $>20 \mu \mathrm{g} / 1$ leading to a significant increase compared with the control group $(\mathrm{P}<0.05)$ (Fig. 1).

A549 cell EMT by $\beta$-catenin. The main factor involved in the classical Wnt signaling pathway is $\beta$-catenin. To examine the role of $\beta$-catenin in the regulation of alveolar EMT, the present study used $\beta$-catenin plasmid-transfected A549 cells. The morphology of the A549 cells changed from a round, cube or polygon shape to a fibroblast-like, stretched, spindle-shape on visualization 


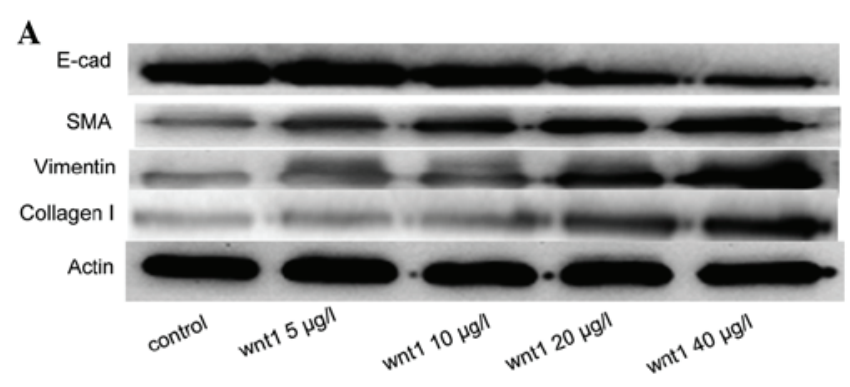

$\mathbf{a}$

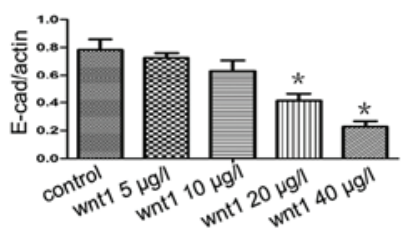

b

c

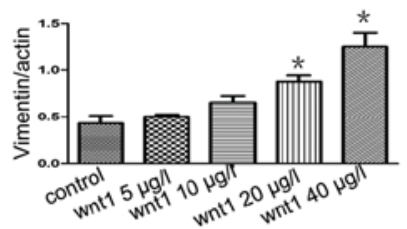

d

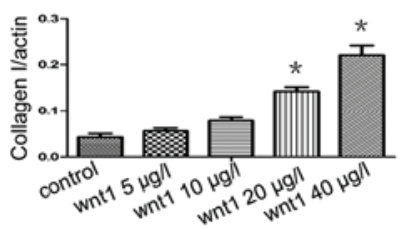

B

a

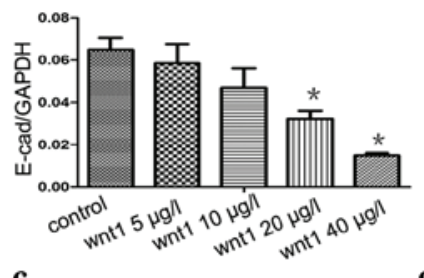

b

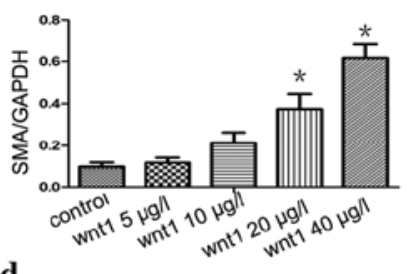

$d$
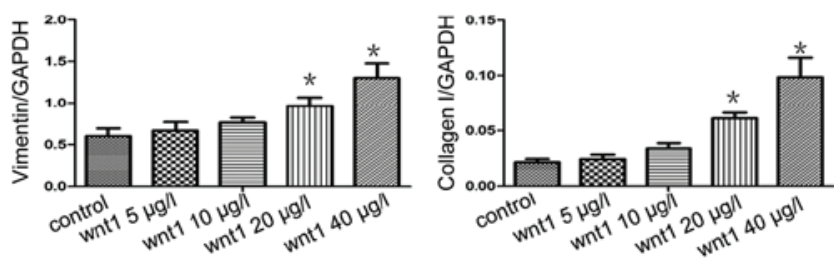

Figure 1. Increased expression of mesenchymal markers and decreased expression of epithelial markers following stimulation of A549 cells with various concentrations of Wnt1. (Aa) Western blot analysis of the protein expression of E-cad. (Ba) RT-qPCR for the mRNA expression of E-cad. (Ab-d) Expression levels of $\alpha$-SMA, vimentin and collagen I protein. (Bb-d) RT-qPCR of the mRNA expression levels of $\alpha$-SMA, vimentin and collagen I. Each bar represents the mean \pm standard deviation; ${ }^{*} \mathrm{P}<0.05$ compared with the control. RT-qPCR, reverse transcription quantitative polymerase chain reaction; E-cad, E-cadherin; $\alpha$-SMA, $\alpha$-smooth muscle actin.

with an inverted phase contrast microscope (Fig. 2A). No changes in morphology was observed in the A549 cells in the control group, which maintained a typical epithelial morphology (polygonal/cobblestone or round appearance; Fig. 2B). In the $\beta$-catenin plasmid group, the relative gene and protein levels of the characteristic epithelial phenotypic marker E-cadherin were significantly lower $(\mathrm{P}<0.05$; Fig. $2 \mathrm{Ca}$ and $\mathrm{Da})$ and the relative expression of the mesenchymal markers $\alpha$-SMA, vimentin and collagen I were significantly higher $(\mathrm{P}<0.05)$ compared with

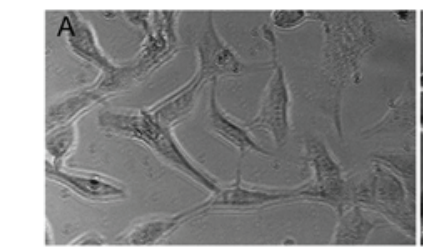

$\beta$-catenin plasmid transfection

C

a

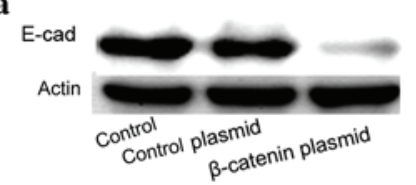

b
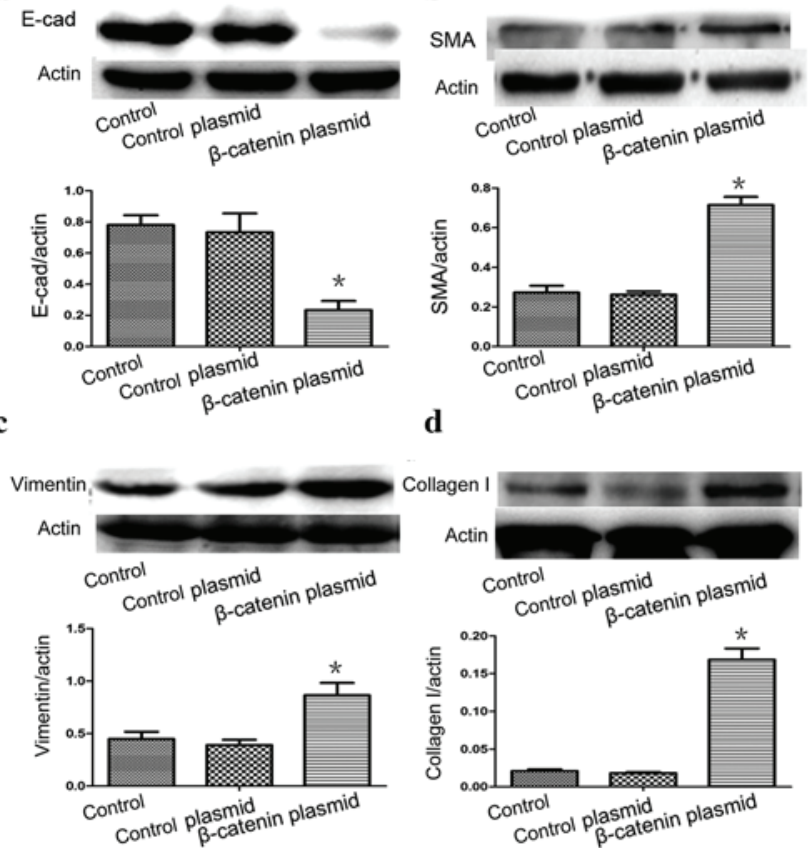

d
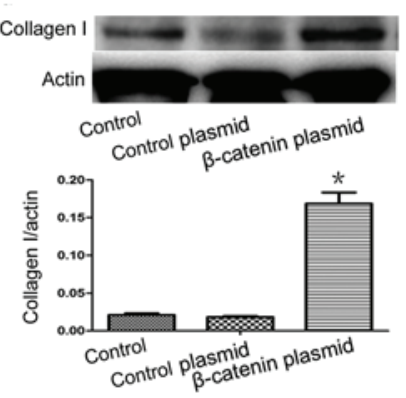

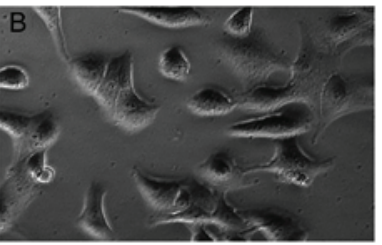

Control b
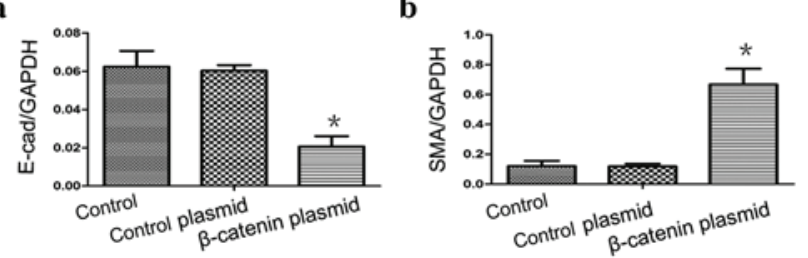

d

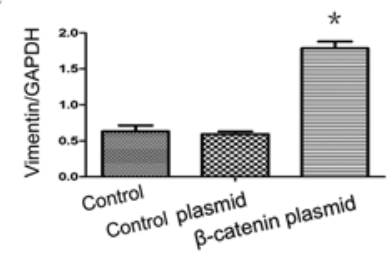

D

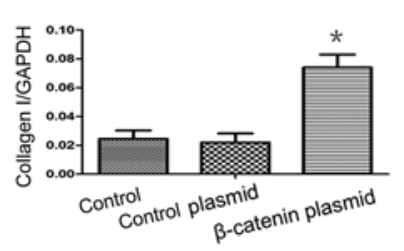

Figure 2. (A) A549 cells were transfected with $\beta$-catenin plasmids and, after $48 \mathrm{~h}$, the A549 cells assumed a fibroblast-like morphology. (B) In the controls, epithelial cells maintained their typical polygonal/cobblestone or round appearance (original magnification, x200). (C) Effects of $\beta$-catenin on the protein expression of E-cad, $\alpha$-SMA, vimentin and collagen $I$ in the A549 epithelial cells. (D) Reverse transcription-quantitative polymerase chain reaction revealed the mRNA expression levels of E-cad, $\alpha$-SMA, vimentin and collagen I following $\beta$-catenin plasmid transfection. (Control, medium-treated A549 cells; control plasmid, A549 cells treated with empty plasmids). Each bar represents the mean \pm standard deviation. ${ }^{*} \mathrm{P}<0.05$, compared with the control. E-cad, E-cadherin; $\alpha$-SMA, $\alpha$-smooth muscle actin.

the control group (Fig. 2Cb-d and Db-d). However, no significant differences were observed in the levels of E-cadherin, $\alpha$-SMA, vimentin or collagen I levels between the empty plasmid group and the control $(\mathrm{P}>0.05)$. Taken together, these 


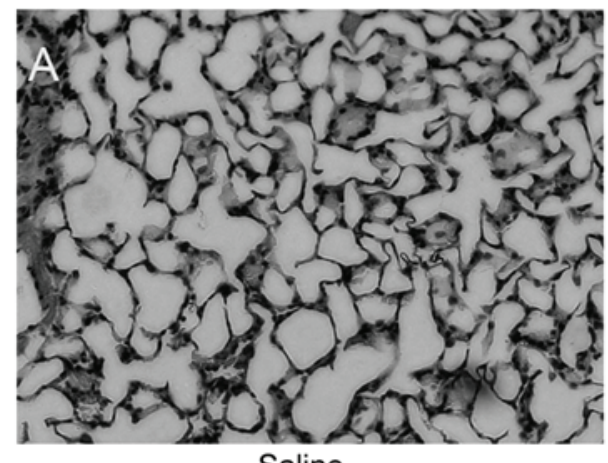

Saline

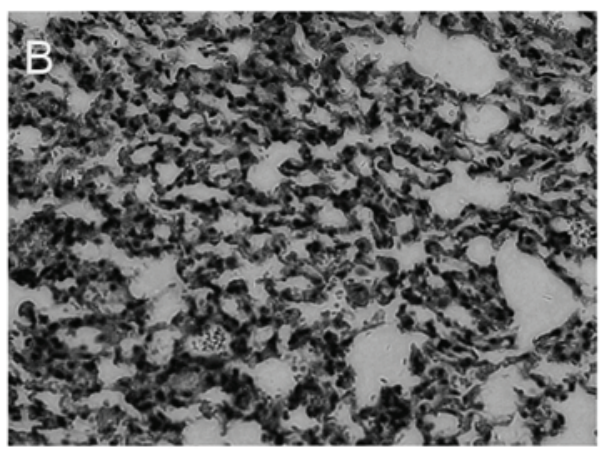

Bleomycin

C

a

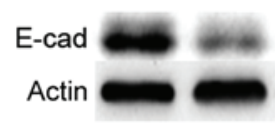

control BALF

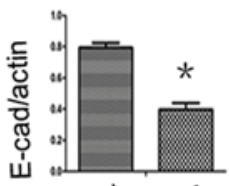

control BALF b

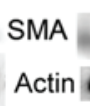

control BALF

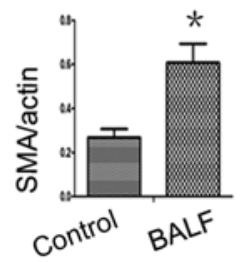

b c d e

D

a

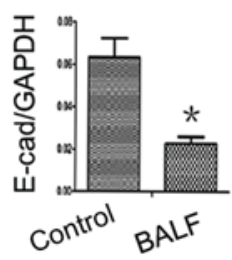

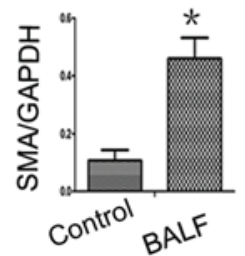

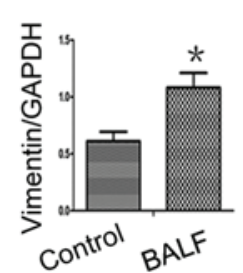

d

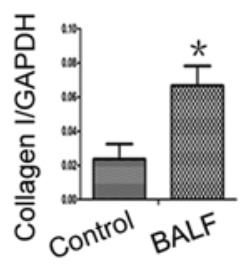

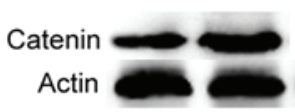

control BALF

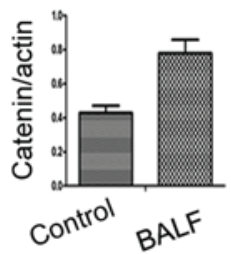

e

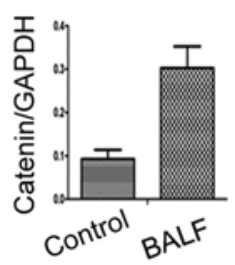

Figure 3. BALF induced a significant reduction in the expression of E-cad and significant increases in the expression levels of $\alpha$-SMA, vimentin and collagen I in the A549 cells. Hematoxylin and eosin-stained lung sections from mice. (A) Control group (saline); (B) BLM group (day 7 post-bleomycin instillation). (C) Protein expression of E-cad, $\alpha$-SMA, vimentin and collagen I, in A549 epithelial cells cultured with BALF. (D) Reverse transcription quantitative polymerase chain reaction of the mRNA expression levels of E-cad, $\alpha$-SMA, vimentin and collagen I in the A549 epithelial cells cultured with BALF. (Ce and De) Changes in the protein and mRNA expression of $\beta$-catenin in BALF-treated A549 cells. (Control, medium-treated A549 cells). Each bar represents means \pm standard deviation; " $\mathrm{P}<0.05$ compared with control. E-cad, E-cadherin; $\alpha$-SMA, $\alpha$-smooth muscle actin; BALF, bronchoalveolar lavage fluid; BLM, bleomycin.

results demonstrated that A549 cells undergo EMT in vitro when exposed to $\beta$-catenin stimuli.

$B A L F$ induces a significant reduction in the expression of $E$-cadherin and significant increases in the expression of $\alpha-S M A$, vimentin and collagen I in A549 cells. At present, the most frequently used experimental model of lung fibrosis is the bleomycin-induced model. In the present study BALF and lung biopsies were obtained from bleomycin-treated mice at day 7. In the H\&E-stained sections, inflammatory cells and erythrocytes were observed in the septum and alveolus, which was associated with fibroblast proliferation (Fig. 3B), indicating successful construction of the bleomycin-induced model of pulmonary fibrosis.

To determine whether lung alveolar epithelial cell injury induced the expansion of the fibroblast and myofibroblast population through EMT, A549 cells were cultured with
BALF and DMEM (1:1) for $48 \mathrm{~h}$ and BALF was obtained from the bleomycin-treated mice at day 7. In the A549 cells, expression of the epithelial phenotypic marker E-cadherin was lost (Fig. 3Ca and Da) and overexpression of $\alpha$-SMA (Fig. 3Cb and Db), vimentin (Fig. 3Cc and Dc) and collagen I (Fig. 3Cd and Dd) were observed by western blot analysis and RT-qPCR. These results indicated the occurrence of a mesenchymal cell phenotype transition, which was absent in the control group $\left({ }^{*} \mathrm{P}<0.05\right)$. Notably, the reduced levels of mRNA and protein expression of E-cadherin correlated with levels of $\beta$-catenin $(r=-0.817$ and -0.831$)$ and the increased levels of mRNA and protein expression of $\alpha$-SMA correlated with levels of $\beta$-catenin $(r=0.825$ and 0.820$)$. The mRNA and protein expression levels of vimentin and collagen I also correlated with $\beta$-catenin levels $(r=0.815$ and 0.816 and $r=0.846$ and 0.831 , repectively). Furthermore, the present study knocked down the $\beta$-catenin gene by infecting the A549 cells with a 
A
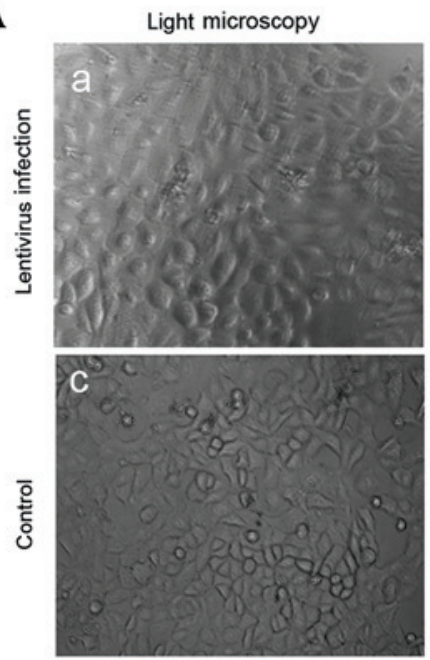

Fluorescence microscopy
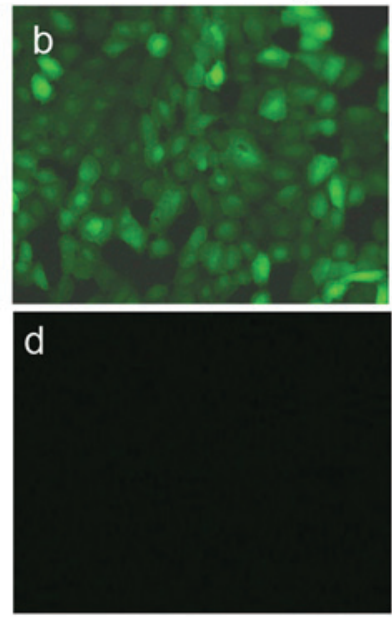

B

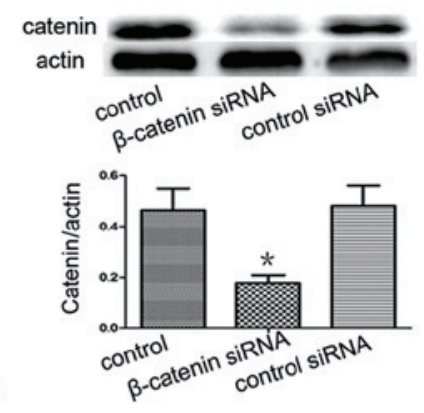

C

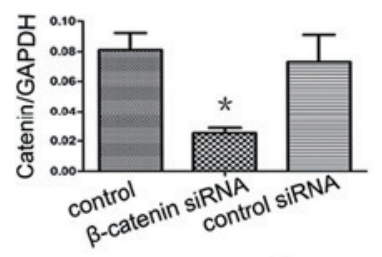

$\mathbf{a}$
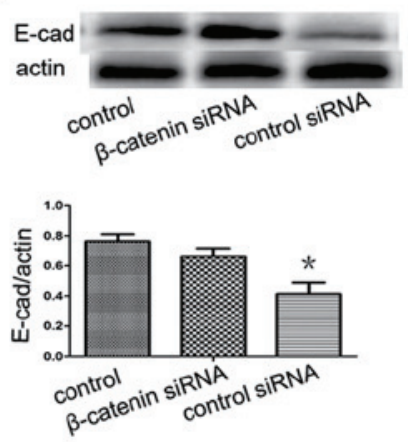

E

a

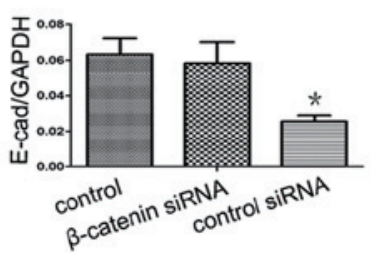

b
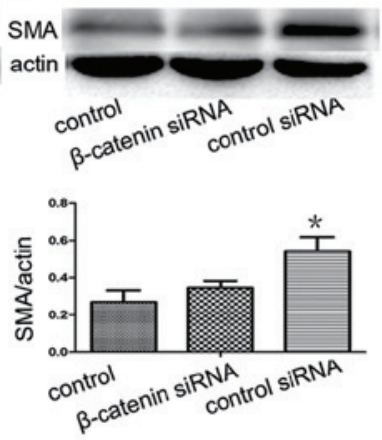

b

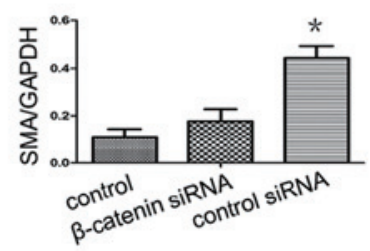

c
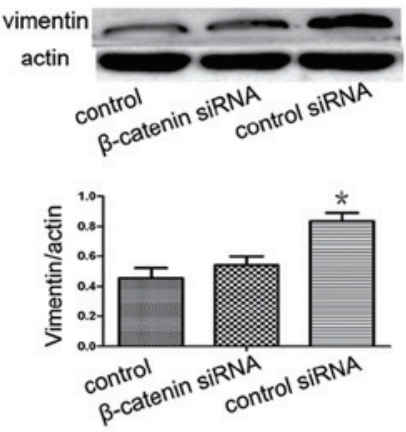

c

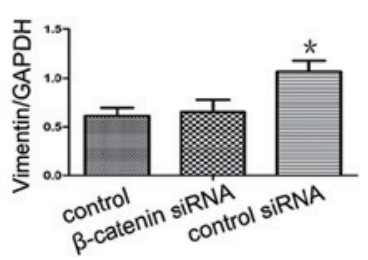

d collagen I actin control $\beta$-catenin sirNA control sirNA

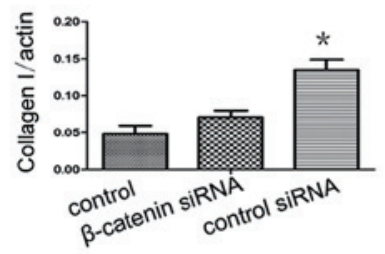

d

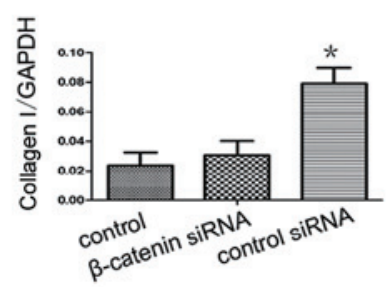

Figure 4. $\beta$-catenin siRNA inhibits the expression of $\beta$-catenin and attenuates epithelial-mesenchymal transition by BALF. (Aa) A549 cells were observed under light microscope $96 \mathrm{~h}$ after infection with lentivirus (x200). (Ab) GFP fluorescence (right panel) of A549 cells was observed using fluorescence microscopy $96 \mathrm{~h}$ after infection with the lentivirus containing pLL-sh catenin or shNC (x200). (Ac) A549 cells were observed under a light microscope with no lentivirus infection. (Ad) GFP fluorescence was not observed when the cells were not infected with the lentivirus. Western blot analysis and RT-qPCR assessment revealed that $\beta$-catenin siRNA suppresses (B) $\beta$-catenin protein and (C) mRNA expression in the A549 cells. Following infection of the A549 cells with the $\beta$-catenin-expressing shRNA lentivirus and BALF treatment, (D) western blot analysis revealed the protein expression of E-cad, $\alpha$-SMA, vimentin and collagen I. (E) mRNA expression of E-cad, $\alpha$-SMA, vimentin and collagen I by RT-qPCR. (Control, medium-treated A549 cells) Each bar represents the mean \pm standard deviation, ${ }^{*} \mathrm{P}<0.05$. GFP, green fluorescent protein; E-cad, E-cadherin; $\alpha$-SMA, $\alpha$-smooth muscle actin; RT-qPCR, reverse transcription quantitative polymerase chain reaction.

lentivirus (sh $\beta$-catenin) expressing $\beta$-catenin-specific siRNA and GFP. After $96 \mathrm{~h}$, the cells expressed GFP (Fig. 4Ab), indicating successful infection. Western blot analysis and RT-qPCR revealed that the levels of $\beta$-catenin in the A549 siRNA-infected cells were significantly lower compared with the cells infected with shNC (Fig. 4B and C). These findings indicated that siRNA, directed towards A549, was effective in specifically knocking down the $\beta$-catenin gene. The A549 cells were then infected with the $\beta$-catenin-expressing shRNA lentivirus prior to BALF treatment. As a negative control, a group of A549 cells were infected by a lentivirus containing an unrelated shRNA sequence. Notably, the siRNA restored the decreased expression level of E-cadherin and increased expression levels of $\alpha$-SMA, vimentin and collagen I that were induced by BALF treatment (Fig. 4D and E).

\section{Discussion}

The Wnt/ $\beta$-catenin signaling pathway is important in the regulation of cell proliferation, differentiation and polarity. Accumulating evidence from animal models and human diseases indicate that Wnt signaling is enhanced in several fibrotic diseases and in lung fibroblasts. Our previous studies demonstrated high expression levels of $\beta$-catenin in bleo- 
mycin-induced pulmonary fibrosis in mice and improvement in pulmonary fibrosis following inhibition of the classical Wnt signaling pathway by SFRP4 antagonists.

In the classical Wnt/ $\beta$-catenin pathway, a complex between the Wnt ligands and the cell surface receptor frizzled (FZD) binds low-density lipoprotein receptor-related protein (LRP), which leads to activation of the dishevelled protein (Dvl), inhibiting phosphorylation of GSK-3 $\beta$ and $\beta$-catenin decomposition. This leads to subsequent $\beta$-catenin translocation into the nucleus, which binds to the transcription factor (TCF)/lymphoid enhancer factor (LEF) and activates target genes and the induction of fibrosis (23). $\beta$-catenin has a dual role. In normal cells, it is located on the cell membrane as a structural protein in connection with E-cadherin that is important in cellular adhesion junctions. On activation of Wnt signaling, $\beta$-catenin, as an intermediary, is translocated into the nucleus (24).

The formation of fibroblastic foci is considered to be the main feature of IPF. Fibroblastic foci are composed mainly of fibroblasts and myofibroblasts. EMT may be an important mechanism in increasing the myofibroblast pool. E-cadherin is a recognized phenotypic marker of epithelial cells, $\alpha$-SMA and collagen I are key markers of a myofibroblast phenotype and vimentin is a cytoskeletal protein. In addition, $\alpha$-SMA and vimentin are often described as mesenchymal cell markers.

In the present study, different concentrations of Wnt1-intervented A549 cells were used. The results demonstrated downregulation in the epithelial phenotypic marker E-cadherin and upregulation of the mesenchymal phenotypic marker and, when the concentration of Wnt1 exceeded $20 \mu \mathrm{g} / \mathrm{l}$, these changes were more obvious. Furthermore, the A549 cells were transfected with a $\beta$-catenin plasmid, which induced a decrease in the mRNA and protein expression levels of E-cadherin and an increase in mRNA and protein expression levels of $\alpha$-SMA, vimentin and collagen I.

Intratracheal bleomycin instillation causes initial alveolar epithelial cell injury and apoptosis (25). Bleomycin-induced injury is widely used as a model of pulmonary fibrosis $(26,27)$. Lewis et al (28) compared different mouse models of infection, allergy and lung injury and found that regulation of the Wnt signaling pathway is specific to the mouse model of bleomycin-induced lung fibrosis. Our previous studies involving bleomycin-induced pulmonary fibrosis in mice demonstrated that the expression of $\beta$-catenin increases on day 7 and peaks on day 14 and that alveolar epithelial injury is most marked on day 7. In the present study, day 7 of BALF was selected in a bleomycin mouse model and the results demonstrated that increases in the mRNA and protein expression of vimentin, $\alpha$-SMA and collagen I were positively correlated with the expression of $\beta$-catenin, however, decreases in the mRNA and protein expression of E-cadherin were negatively correlated with the expression of $\beta$-catenin. Furthermore, the present study also infected A549 cells with a lentivirus containing $\beta$-catenin shRNA, which knocked down the $\beta$-catenin gene, and the lung epithelial cells were then cultured with the pulmonary lavage fluid. Following this, no significant increases were observed in the mRNA and protein expression levels of vimentin, $\alpha$-SMA and collagen I and no decrease was observed in the mRNA and protein expression levels of E-cadherin. The expression of
Wnt ligands and $\beta$-catenin in the pulmonary lavage fluid from mice in the bleomycin model were not measured in the present study, however, Levänen et al (29) observed that the mRNA expression levels of Wnt5A, Wnt7A and Wnt7B increased in BALF cells in patients with sarcoidosis. A possible mechanism for this may be that bleomycin-induced epithelial injury triggers an acute inflammatory response and initiates lung repair mechanisms, including activation of the Wnt signaling pathway. The Wnt family proteins are released by the injured epithelial cells and neighboring cells to the surrounding tissues and into the BALF. In the present study, the use of BALF in the A549 cell culture, led to $\beta$-catenin nuclear transcription, binding to TCF/LEF and activation of downstream target genes, inducing cell EMT even in the absence of initial injury factors. TGF- $\beta$ is considered to be a key mediator in the progression of fibrosis (30). Previous studies have demonstrated that there are cross talks between the Wnt/ $\beta$-catenin pathway and TGF- $\beta$ signaling (31-33). In bleomycin-induced mice, protein levels of TGF- $\beta$ in BALF are significantly increased $(34,35)$. In the present study, it was hypothesized that the BALF obtained from the bleomycin-induced pulmonary fibrosis mouse model contained TGF- $\beta$, which activated the Wnt signal through cross talk with the $\mathrm{Wnt} / \beta$-catenin pathway.

The BALF from pulmonary fibrosis mouse models contains interleukin (IL)-1 $\alpha$, IL-6 and tumor necrosis factor (TNF)- $\alpha$ (36). Whether the Wnt signal is triggered by IL-1 $\alpha$, IL-6 or TNF- $\alpha$ requires further investigation.

In conclusion, the present study demonstrated that Wnt/ $\beta$-catenin signaling increases the number of myofibroblasts in pulmonary fibrosis through EMT. In addition, it revealed that activation of the biological repair response at an injury site and its persistence is important in the formation of pulmonary fibrosis. In lung injury, the reactivation of aberrant Wnt/ $\beta$-catenin signaling is important in the formation of fibrotic diseases and may provide a potential therapeutic strategy in the future.

\section{Acknowledgements}

This study was supported by grants from the Scientific Research Project of the Ministry of Public Health (no. wkj2006-2-026) and the Shanghai Science and Technology Development Fund (no. 10ZR1422600).

\section{References}

1. Kliment CR, Englert JM, Gochuico BR, et al: Oxidative stress alters syndecan-1 distribution in lungs with pulmonary fibrosis. J Biol Chem 284: 3537-3545,2009.

2. Selman M and Pardo A: The epithelial/fibroblastic pathway in the pathogenesis of idiopathic pulmonary fibrosis. Am J Resp Cell Mol Bio 29: S93-S97, 2003.

3. Ramos C, Becerril C, Montaño M, et al: FGF-1 reverts epithelial-mesenchymal transition induced by TGF- $\{$ beta $\} 1$ through MAPK/ERK kinase pathway. Am J Physiol Lung Cell Mol Physiol 299: L222-L231, 2010.

4. Willis BC, Liebler JM, Luby-Phelps K, et al: Induction of epithelial-mesenchymal transition in alveolar epithelial cells by transforming growth factor- $\beta 1$ : potential role in idiopathic pulmonary fibrosis. Am J Pathol 166: 1321-1332, 2005.

5. Kim KK, Kugler MC, Wolters PJ, et al: Alveolar epithelial cell mesenchymal transition develops in vivo during pulmonary fibrosis and is regulated by the extracellular matrix. Proc Natl Acad Sci USA 103: 13180-13185, 2006. 
6. Willis BC and Borok Z: TGF-beta-induced EMT: mechanisms and implications for fibrotic lung disease. AM J Physiol Lung Cell Mol Physiol 293: L525-L534, 2007.

7. Tanjore $\mathrm{H}, \mathrm{Xu} \mathrm{XC}$, Polosukhin VV, et al: Contribution of epithelial-derived fibroblasts to bleomycin-induced lung fibrosis. Am J Respir Crit Care Med 180: 657-665, 2009.

8. Selman M and Pardo A: Idiopathic pulmonary fibrosis: misunderstandings between epithelial cells and fibroblasts? Sarcoidosis Vasc Diffuse Lung Dis 21: 165-172, 2004.

9. Acloque H, Adams MS,Fishwick K, etal: Epithelial-mesenchymal transitions: the importance of changing cell state in development and disease. J Clin Invest 119: 1438-1449, 2009.

10. Kalluri R and Weinberg RA: The basics of epithelial-mesenchymal transition. J Clin Invest 119: 1420-1428, 2009.

11. Kalluri R and Neilson EG: Epithelial-mesenchymal transition and its implications for fibrosis. J Clin Invest 112: 1776-1784, 2003.

12. Zavadil J and Böttinger EP: TGF-beta and epithelial-to-mesenchymal transitions. Oncogene 24: 5764-5774, 2005.

13. Morali OG, Delmas V, Moore R, et al: IGF-II induces rapid $\beta$-catenin relocation to the nucleus during epithelium to mesenchyme transition. Oncogene 20: 4942-4950, 2001.

14. Strutz F, Zeisberg M, Ziyadeh FN, et al: Role of basic fibroblast growth factor-2 in epithelial-mesenchymal transformation. Kidney Int 61: 1714-1728, 2002.

15. Kasai H, Allen JT, Mason RM, et al: TGF- $\beta 1$ induces human alveolar epithelial to mesenchymal cell transition (EMT). Respir Res 6: 56, 2005 .

16. Meuten T, Hickey A, Franklin K, et al: WNT7B in fibroblastic foci of idiopathic pulmonary fibrosis. Respir Res 13: 62, 2012.

17. Guo Y, Xiao L, Sun L and Liu F: Wnt/beta-catenin signaling: a promising new target for fibrosis diseases. Physiol Res 61 : 337-346, 2012

18. Borok Z: Role for alpha3 integrin in EMT and pulmonary fibrosis. J Clin Invest 119: 7-10, 2009.

19. Selman M and Pardo A: Role of epithelial cells in idiopathic pulmonary fibrosis: from innocent targets to serial killers. Proc Am Thorac Soc 3: 364-372, 2006.

20. Lawson WE, Polosukhin VV, Stathopoulos GT, et al: Increased and prolonged pulmonary fibrosis in surfactant protein C-deficient mice following intratracheal bleomycin. Am J Pathol 167: 1267-1277, 2005.

21. Jiang D, Liang J, Campanella GS, et al: Inhibition of pulmonary fibrosis in mice by CXCL10 requires glycosaminoglycan binding and syndecan-4. J Clin Invest 120: 2049-2057, 2010.
22. Jiang D, Liang J, Hodge J, et al: Regulation of pulmonary fibrosis by chemokine receptor CXCR3. J Clin Invest 114: 291-299, 2004

23. Moon RT, Kohn AD, De Ferrari GV and Kaykas A: WNT and $\beta$-catenin signalling: diseases and therapies. Nat Rev Genet 5: 691-701, 2004.

24. Bao XL, Song H, Chen Z and Tang X: Wnt3a promotes epithelial-mesenchymal transition, migration, and proliferation of lens epithelial cells. Mol Vis 18: 1983-1990, 2012.

25. Gauldie J, Bonniaud P, Sime P, et al: TGF-beta, Smad3 and the process of progressive fibrosis. Biochem Soc Trans 35: 661-664, 2007.

26. Moeller A, Ask K, Warburton D, et al: The bleomycin animal model: a useful tool to investigate treatment options for idiopathic pulmonary fibrosis? Int J Biochem Cell Biol 40: 362-382, 2008.

27. Moore BB and Hogaboam CM: Murine models of pulmonary fibrosis. Am J Physiol Lung Cell Mol Physiol 294: L152-L160, 2008.

28. Lewis CC, Yang JY,Huang X, et al: Disease-specific gene expression profiling in multiple models of lung disease. Am J Respir Crit Care Med 177: 376-387, 2008

29. Levänen B, Wheelock AM, Eklund A, et al: Increased pulmonary Wnt (wingless/integrated)-signaling in patients with sarcoidosis. Respir Med 105: 282-291, 2011.

30. Biernacka A, Dobaczewski M and Frangogiannis NG: TGF- $\beta$ signaling in fibrosis. Growth Factors 29: 196-202, 2011.

31. Carre AL, James AW, MacLeod L, et al: Interaction of wingless protein (Wnt), transforming growth factor-beta1, and hyaluronan production in fetal and postnatal fibroblasts. Plast Reconstr Surg 125: 74-88, 2010.

32. Sato M: Upregulation of the Wnt/beta-catenin pathway induced by transforming growth factor-beta in hypertrophic scars and keloids. Acta Derm Venereol 86: 300-307, 2006.

33. Cheon SS, Nadesan P, Poon R and Alman BA: Growth factors regulate beta-catenin-mediated TCF-dependent transcriptional activation in fibroblasts during the proliferative phase of wound healing. Exp Cell Res 293: 267-274, 2004.

34. Izumo T, Kondo M and Nagai A: Effects of a leukotriene B4 receptor antagonist on bleomycin-induced pulmonary fibrosis. Eur Respir J 34: 1444-1451, 2009.

35. Robb WB, Condron C, Moriarty M, et al: Taurine attenuates radiation-induced lung fibrosis in $\mathrm{C} 57 / \mathrm{B} 16$ fibrosis prone mice. Ir J Med Sci 179: 99-105, 2010.

36. Jiang C, Huang H, Liu J, et al: Fasudil, a rho-kinase inhibitor, attenuates bleomycin-induced pulmonary fibrosis in mice. Int $\mathrm{J}$ Mol Sci 13: 8293-8307, 2012. 